\title{
Predictors of Incident Mild Cognitive Impairment and Its Course in a Diverse Community-Based Population
}

\author{
Milou J. Angevaare, MD, Jet M.J. Vonk, PhD, Laiss Bertola, PhD, Laura Zahodne, PhD, \\ Caitlin Wei-Ming Watson, MSc, Amelia Boehme, PhD, Nicole Schupf, PhD, Richard Mayeux, MD, \\ Mirjam I. Geerlings, PhD, and Jennifer J. Manly, PhD
}

Neurology ${ }^{\circledR}$ 2022;98:e15-e26. doi:10.1212/WNL.0000000000013017

\author{
Correspondence \\ Dr. Manly \\ jjm71@cumc.columbia.edu
}

\begin{abstract}
Background and Objectives

To investigate sociodemographic and medical predictors of incident mild cognitive impairment (MCI) and subsequent course of $\mathrm{MCI}$ at follow-up, including sustained MCI diagnosis, classification as cognitively normal, and progression to dementia.
\end{abstract}

\section{Methods}

Within a community-based cohort, diagnoses of MCI were made with a published algorithm. Diagnosis of dementia was based on clinical consensus. Cox regressions estimated hazard ratios of incident MCI associated with several predictors. Modified Poisson regressions estimated relative risks associated with predictors of diagnostic status at follow-up after incidence.

\section{Results}

Among 2,903 cognitively normal participants at baseline, 752 developed MCI over an average of 6.3 (SD 4.5) years (incidence rate 56 per 1,000 person-years). Presence of APOE $\varepsilon 4$ and higher medical burden increased risk of incident $\mathrm{MCI}$, while more years of education, more leisure activities, and higher income decreased this risk. Of the incident MCI cases, after an average of 2.4 years of follow-up, $12.9 \%$ progressed to dementia, $9.6 \%$ declined in functioning and did not meet the algorithmic criteria for MCI but did not meet the clinical criteria for dementia, 29.6\% continued to meet $\mathrm{MCI}$ criteria, and $47.9 \%$ no longer met MCI criteria. Multidomain MCI, presence of $A P O E \varepsilon 4$, depressive symptoms, and antidepressant use increased the risk of progression to dementia.

\section{Discussion}

This community-based study showed that almost half of the individuals with incident MCI diagnoses were classified as cognitively normal at follow-up. Predictors of incident MCI demonstrably differed from those of subsequent MCI course; these findings can refine expectations for cognitive and functional course of those presenting with MCI.

\section{MORE ONLINE}

CME Course

NPub.org/cmelist

From the Department of Neurology and Taub Institute for Research on Alzheimer's Disease and the Aging Brain (M.J.A., J.M.J.V., L.B., L.Z., C.W.-M.W., A.B., N.S., R.M., J.J.M.), College of Physicians and Surgeons, Columbia University, New York, NY; Julius Center for Health Sciences and Primary Care (M.J.A., J.M.J.V., M.I.G.), University Medical Center Utrecht; Amsterdam UMC (M.J.A.), Vrije Universiteit Amsterdam, Department of Medicine for Older People, Amsterdam Public Health Research Institute, Van der Boechorststraat 7, the Netherlands; and National Institute of Science and Technology in Molecular Medicine (L.B.), Federal University of Minas Gerais, Belo Horizonte, Brazil.

Go to Neurology.org/N for full disclosures. Funding information and disclosures deemed relevant by the authors, if any, are provided at the end of the article. 


\section{Glossary}

DSM-III = Diagnostic and Statistical Manual of Mental Disorders, 3rd edition; $\mathbf{M C I}=$ mild cognitive impairment; WHICAP = Washington Heights-Inwood Columbia Aging Project.

Identifying risk factors of mild cognitive impairment (MCI), a prodromal phase of dementia, in cognitively normal older adults can aid characterization of a target group for prevention or intervention strategies of dementia. ${ }^{1-7}$ However, not everyone who is diagnosed with MCI subsequently progresses to dementia; longitudinal studies have shown that 5\% to 53\% of people identified as having MCI at 1 visit no longer meet MCI criteria at the next visit. ${ }^{4,8-13}$ Thus, identifying risk factors of progression to dementia in individuals diagnosed with incident MCI is equally important to refine the selection of individuals at high risk for dementia. ${ }^{10,14}$

Characterization of MCI is typically based on the Petersen criteria, using cutoff scores for cognitive impairment and daily functioning. ${ }^{9} 10,12 \mathrm{MCI}$ diagnosis can be further characterized by the type (i.e., amnestic vs nonamnestic) and number of cognitive domains affected (i.e., single domain vs multidomain). ${ }^{2}$ While MCI criteria require relatively preserved daily functioning, previous studies have shown that individuals with MCI have more difficulties in activities of daily functioning than cognitively normal individuals, ${ }^{15}$ particularly in case of multidomain $\mathrm{MCI}{ }^{16}$ In turn, multidomain $\mathrm{MCI}$ has been consistently identified as a predictor of progression to dementia. ${ }^{9,10}$

We previously reported predictors of progression in prevalent MCI in a multiethnic community-based cohort. ${ }^{4}$ This study extends these findings by investigating modifiable and nonmodifiable risk factors of incident $\mathrm{MCI}$ among non-Hispanic White, non-Hispanic Black, and Hispanic cognitively normal individuals. We also determine which factors predict progression to dementia or classification as cognitively normal at follow-up in those who developed incident MCI.

\section{Methods}

\section{Standard Protocol Approvals, Registrations, and Patient Consents}

This study was approved by the Columbia University Institutional Review Board, and each participant provided informed consent.

\section{Participants}

The participants for this study were selected from the Washington Heights-Inwood Columbia Aging Project (WHICAP), a longitudinal study of aging and dementia in the community, including non-Hispanic White, non-Hispanic Black, and Hispanic people. Participants were recruited by random sampling of persons $\geq 65$ years of age and eligible for Medicare from 3 census tracts in Northern Manhattan, NY, across 3 waves in 1992, 1999, and 2009 (described in detail elsewhere ${ }^{17}$ ).
Follow-up visits were scheduled every 18 to 24 months. Each visit consisted of a medical evaluation including general and neurologic evaluations and health questionnaires, a standard battery of neuropsychological tests, and questionnaires on socioeconomic factors and functional abilities. The study and sampling methods have been described in more detail previously. ${ }^{18,19}$ At the time of the current study, 6,541 participants had been recruited and seen at a total of 20,036 visits. The current study selected participants using 3 criteria: (1) participants should have all the necessary data for determining MCI status during at least 1 of their visits (the first visit with these data was defined as that participant's baseline visit); (2) participants should be free of MCI or dementia at this baseline visit; and (3) participants should have at least 1 follow-up.

\section{$\mathrm{MCl}$ and Dementia}

The MCI diagnosis was retrospectively applied for each visit independently with the use of a published algorithm by investigators blinded to previous diagnoses and was based on 4 criteria expanded from the Petersen criteria and developed for this ethnically and linguistically diverse cohort. ${ }^{4,18}$ The first criterion is a subjective memory complaint, assessed by a questionnaire. The second criterion is objective cognitive impairment in at least 1 cognitive domain, defined as scoring 1.5 SDs below robust age, years of education, ethnicity, and sex/genderadjusted norms ${ }^{18}$ for a composite score of neuropsychological measures within that domain. The third criterion was preserved daily functioning, quantified as impairment in $<3$ instrumental activities of daily functioning (e.g., using the phone, shopping, and handling own medication) based on self-report or observer report. ${ }^{20}$ The fourth criterion was no consensus diagnosis of dementia at that visit. Dementia diagnosis was considered at a consensus conference with neurologists and neuropsychologists present from clinical and neuropsychological data using the DSM-III criteria (revised) ${ }^{21}$ (diagnostic procedures described in detail elsewhere $\left.{ }^{4,18}\right)$.

Four subtypes of MCI were defined on the basis of domain(s) of objective cognitive impairment: single-domain amnestic $\mathrm{MCI}$, single-domain nonamnestic (executive, language, or visuospatial) $\mathrm{MCI}$, multidomain amnestic $\mathrm{MCI}$, and multidomain nonamnestic MCI.

\section{Demographic Factors}

Demographic factors (age, sex/gender, years of education, race/ethnicity) were collected at baseline. Race and Hispanic ethnicity were determined via self-report using the format of the 1990 and 2000 US census, in which participants were asked (1) to classify themselves racially (choose any that apply: White, Black, Asian, American Indian, Pacific Islander, or other) and (2) if they were of Hispanic origin. ${ }^{4,18,22}$ 


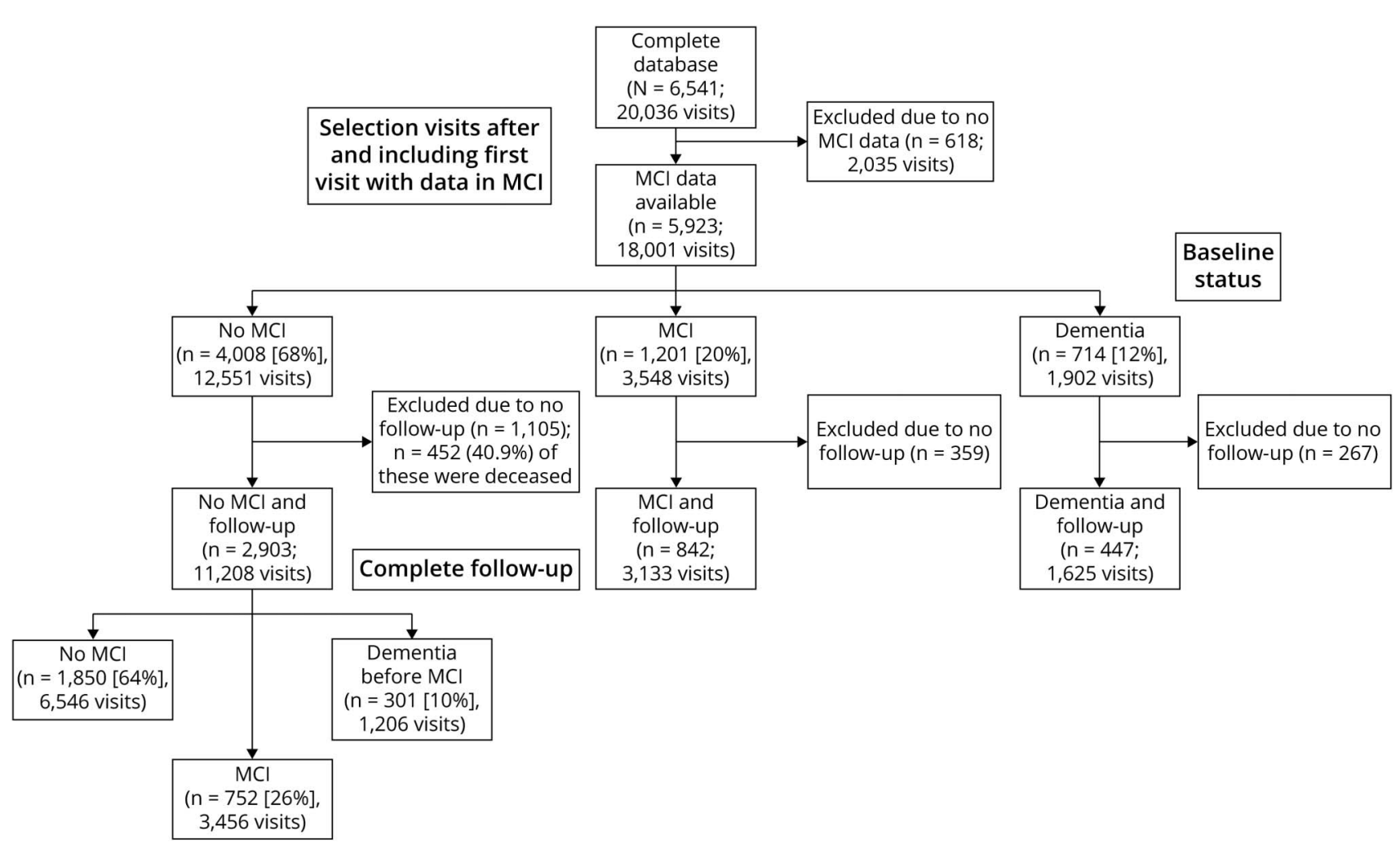

$\mathrm{MCl}=$ mild cognitive impairment.

Subsequently, the WHICAP study categorized race/ethnicity into 3 groups: non-Hispanic White, non-Hispanic Black, and Hispanic (of any race).We use the term sex/gender because participants were asked if they are male or female. This assessment did not allow us to determine whether biological sex or gender was reported. ${ }^{23}$

\section{Socioeconomic Factors}

Primary lifetime occupation was assessed by self-report at baseline. For the current analyses, occupation was grouped into 3 training/skill levels: low (housewives and unskilled workers), medium (skilled and office workers), or high (managers, professionals, and technical occupations). Household income was categorized into low $(<\$ 9,000 / y)$, medium $(\$ 9,000-\$ 36,000 / y)$, or high $(>\$ 36,000 / y)$. Participants were asked about marital status at each visit; a dichotomous variable was created to indicate whether the participant was married. Participation in 13 separate leisure activities was assessed by self-report at each visit and analyzed as a sum score. ${ }^{24}$

\section{Medical and Genetic Factors}

Self-reported medical conditions were collected by a trained medical interviewer; an index of illness burden was calculated as the sum score of the presence of 15 chronic somatic conditions. ${ }^{25}$ APOE genotyping in WHICAP participants has been described in detail previously ${ }^{26}$; participants were classified as either carrier (homozygous or heterozygous) or noncarrier of the $A P O E$ \&4 allele.

Depressive Symptoms and Antidepressant Use Depressive symptoms were assessed at each visit with the 10item version of the Center for Epidemiological Studies Depression Scale. ${ }^{27}$ For the analyses, a dichotomous variable indicating significant depressive symptoms using the conventional cutoff score of 4 was created. Participants were asked to provide a current list of medications or their medicine bottles at each visit, allowing current use of any class of antidepressants to be recorded.

\section{Statistical Analysis}

\section{Incident $\mathbf{M C l}$}

Participants were classified into 3 groups depending on their diagnostic status throughout the follow-up period: (1) those who remained cognitively normal throughout follow-up, (2) those with incident MCI, and (3) those who progressed to dementia directly from cognitively normal without an intermediate diagnosis of MCI (Figure 1). Incidence rates of MCI and the 4 MCI subtypes (i.e., single-domain amnestic MCI, single-domain nonamnestic MCI, multidomain amnestic MCI, and multidomain nonamnestic MCI) were calculated per 1,000 person-years by dividing the incidence rate by the length of follow-up; incidence rates and $95 \%$ confidence intervals were based on a Poisson distribution. 
Cox proportional hazards models were used to identify predictors of incident $\mathrm{MCI}$, including education, sex/gender, race/ethnicity, occupation, $A P O E \varepsilon 4$ status, marital status, medical burden, leisure activity, income, antidepressant use, and depressive symptoms. First, we estimated a series of models that tested each predictor individually, adjusting for age at baseline and recruitment wave. Subsequently, we tested a full model that included all predictors. Due to interval censoring, the onset of $\mathrm{MCI}$ was set at the midpoint between the last visit with normal cognition and the first visit with $\mathrm{MCI}$ or the first visit with a dementia diagnosis in those cases in which dementia diagnosis was not preceded by MCI. Participants who did not develop MCI or dementia were censored at the time of their last visit. Censoring includes loss of followup due to death or withdrawal; censoring was based on the last visit, whether a participant was still taking part in further follow-ups, or whether there had been an end of follow-up due to death or other reasons.

We performed a sensitivity analysis of the full model to assess mortality as a competing risk because of the likely association between development of MCI and death. ${ }^{28}$ For this analysis, all those who were lost to follow-up due to death were hypothetically assumed to have died with MCI. Time to (hypothetical) MCI was calculated as the midpoint between date of the last visit and date of death. We compared the hazard ratios of this sensitivity analysis with those of the main analysis; similar hazard ratios would suggest noninformative censoring in case of death.

Data were missing for 7 of the predictor variables for these analyses, ranging from $<1 \%$ to $24 \%$ per variable (Table 1 ). The reasons for missing data in most cases were changes in the assessment protocol over time and the recruitment of new cohorts, which resulted in some variation in measures across visits and recruitment waves. For example, the Center for Epidemiological Studies Depression Scale was first implemented in 1999, so only 1992 cohort participants who were seen after 1999 were administered that measure. We used multiple imputation to account for missing data, using pooled estimates from 10 imputations based on a fully conditional specification imputation method with 10 iterations.

\section{Course of $\mathrm{MCl}$}

Only participants with at least 1 follow-up visit after diagnosis of incident MCI were included to analyze the course of MCI (i.e., status at the first follow-up visit after incident MCI) (Figure 2). Descriptive statistics were used to compare participants with and without a follow-up visit after incident $\mathrm{MCI}$ diagnosis.

We used modified Poisson regression (i.e., Poisson regression with a robust error variance) to estimate relative risks for predictors for each diagnostic category (i.e., sustained MCI diagnosis, progression to dementia, or functional decline, an additional outcome category that was identified during analysis) compared to those who did not meet cognitive criteria for MCI at follow-up. For prospective studies, odds ratios (obtained in a logistic model) overestimate relative risk if the outcome is frequent, as was the case in this study; therefore, we used modified Poisson regression to estimate relative risks. ${ }^{29}$

Predictor variables included education, sex/gender, race/ ethnicity, occupation, $A P O E \varepsilon 4$ status, marital status, medical burden, leisure activity, income, antidepressant use, depressive symptoms, and single-domain vs multidomain incident MCI status. Due to a smaller number of participants available in this analysis compared to the analysis of incident $\mathrm{MCI}$, the medium and high groups of income and occupation were combined. Models were adjusted for age at incident MCI and recruitment wave. Because the diagnostic status at follow-up after incident MCI may depend on time of follow-up, the natural log transformation of time to follow-up in months (i.e., months between incident and first follow-up after incidence) was used as an offset variable. First, we estimated a series of models that tested each predictor individually, adjusting for age and recruitment wave. Subsequently, we tested a full model that included all predictors. We used multiple imputation to account for missing data, incorporating pooled estimates from 10 imputations based on a fully conditional specification imputation method with 10 iterations. IBM SPSS Statistics version 25 (Armonk, NY) was used for all analyses. ${ }^{30}$

\section{Data Availability}

Data are available on reasonable request to the WHICAP Publications Committee. Data requests should be submitted at cumc.col.qualtrics.com/jfe/form/SV_6x5rRy14B6vpoqN.

\section{Results}

\section{Incident $\mathrm{MCl}$}

The study sample consisted of 2,903 participants with 11,208 visits (Figure 1) and an average of 3.7 (SD 1.8) visits over 6.3 (SD 4.5) years of follow-up. Table 1 shows the baseline characteristics for participants without incident $\mathrm{MCI}$, those with incident MCI, and those who progressed to dementia without a diagnosis of MCI. Table 2 provides incidence rates of $\mathrm{MCI}$ in general and per MCI subtype.

Table 3 shows the results of the Cox regression analysis of predictors of incident MCI. In a series of models that examined the individual relationships between each predictor and incident MCI, we observed higher risk of incident MCI with being of Hispanic ethnicity, being an APOE $\varepsilon 4$ carrier, having higher medical burden, and having more depressive symptoms, and a lower risk of incident MCI was associated with more years of education, higher level of occupation, more leisure activities, and higher income. However, when all predictors were entered into the full model together, ethnic background, occupation, and depressive symptoms dropped out of the model; that is, $A P O E \varepsilon 4$ allele and higher medical burden were associated with a higher risk of incident $\mathrm{MCI}$, while more years of education, more leisure activities, and higher income were associated with lower risk of incident MCI. 
Table 1 Baseline Participant Characteristics Among Diagnostic Groups (Before Multiple Imputation) and Distribution of Variables Among Participants Without and With Missing Values

\begin{tabular}{|c|c|c|c|c|c|}
\hline \multirow[b]{2}{*}{ Diagnostic group } & \multicolumn{3}{|c|}{$\begin{array}{l}\text { Baseline participant characteristics among } \\
\text { diagnostic groups }\end{array}$} & \multicolumn{2}{|c|}{$\begin{array}{l}\text { Distribution of variables among participants } \\
\text { without and with missing values }\end{array}$} \\
\hline & $\begin{array}{l}\text { No incident } \\
\mathrm{MCl} / \text { dementia }\end{array}$ & Incident $\mathrm{MCI}$ & $\begin{array}{l}\text { Incident } \\
\text { dementia }\end{array}$ & Data complete & Data incomplete \\
\hline No. (\%) & $1,850(63.7)$ & $752(25.9)$ & $301(10.4)$ & $1,627(56)$ & $1,276(44)$ \\
\hline Age, mean (SD), y & $74.7(6.0)$ & $76.1(6.0)$ & $79.5(7.1)$ & $74.9(6.2)$ & $76.3(6.3)$ \\
\hline Education, mean (SD), y & $11.5(4.8)$ & $9.88(4.8)$ & $7.01(4.5)$ & $10.9(5.0)$ & $10.2(4.9)$ \\
\hline Follow-up, mean (SD), y & $5.43(4.1)$ & $8.12(4.8)$ & $6.7(4.6)$ & $5.50(3.78)$ & $7.22(5.17)$ \\
\hline \multicolumn{6}{|l|}{ Cohort, n (\%) } \\
\hline 1992 & $340(18.4)$ & $242(32.2)$ & $112(37.2)$ & $1(0.1)$ & $693(54.3)$ \\
\hline 1999 & $674(36.4)$ & 345 (45.9) & $118(39.2)$ & $724(44.5)$ & $413(32.4)$ \\
\hline 2009 & $836(45.2)$ & 165 (21.9) & $71(23.6)$ & $902(55.4)$ & $170(13.3)$ \\
\hline Women, n (\%) & $1,242(67.1)$ & $520(69.1)$ & $216(71.8)$ & $1,089(66.9)$ & $889(69.7)$ \\
\hline Black, n (\%) & $582(31.5)$ & $228(30.3)$ & $79(26.2)$ & 477 (29.3) & $412(32.3)$ \\
\hline Hispanic, n (\%) & $713(38.5)$ & $328(43.6)$ & $192(63.8)$ & $755(46.4)$ & $478(37.5)$ \\
\hline \multicolumn{6}{|l|}{ Occupation, n (\%) } \\
\hline Low & $719(41.1)$ & $408(56.8)$ & $215(75.2)$ & $749(46.0)$ & $593(52.7)$ \\
\hline Medium & $461(26.4)$ & $172(24.0)$ & $46(16.1)$ & $374(23.0)$ & $305(27.1)$ \\
\hline High & $569(32.5)$ & $138(19.2)$ & $25(8.7)$ & $504(31.0)$ & $228(20.2)$ \\
\hline APOE \&4 carrier, $\mathrm{n}$ (\%) & $459(25.6)$ & $195(26.7)$ & $86(30.2)$ & $428(26.3)$ & $312(26.5)$ \\
\hline Married, n (\%) & $614(35.1)$ & $207(30.4)$ & $76(27.7)$ & $557(34.2)$ & $340(31.7)$ \\
\hline Medical burden, mean (SD) & $2.09(1.6)$ & $2.00(1.5)$ & $2.31(1.6)$ & $2.40(1.6)$ & $1.69(1.5)$ \\
\hline Leisure activities, mean (SD) & $7.50(2.5)$ & $7.40(2.4)$ & $5.78(2.5)$ & $7.39(2.5)$ & $7.17(2.6)$ \\
\hline \multicolumn{6}{|l|}{ Income, $\mathbf{n} \%$} \\
\hline Low & $433(27.4)$ & $281(44.1)$ & $153(61.7)$ & $425(26.1)$ & $442(52.9)$ \\
\hline Medium & $860(54.5)$ & $301(47.3)$ & $83(33.5)$ & $908(55.8)$ & $336(40.2)$ \\
\hline High & $285(18.1)$ & $55(8.6)$ & $12(4.8)$ & $294(18.1)$ & $58(6.9)$ \\
\hline Antidepressant use, n \% & $127(7.3)$ & $41(6.0)$ & $20(7.1)$ & $143(8.8)$ & $45(4.1)$ \\
\hline Depressive symptoms, $\mathrm{n} \%$ & $232(15.4)$ & $85(16.6)$ & $49(26.1)$ & $256(15.7)$ & $110(18.9)$ \\
\hline Event, $n(\%)$ & - & - & - & $491(30.2)$ & $562(44)$ \\
\hline Time to event, mean (SD), y & - & - & - & $4.39(3.5)$ & $4.94(4.5)$ \\
\hline
\end{tabular}

Abbreviation: $\mathrm{MCl}=$ mild cognitive impairment

Participant characteristics based on data before imputation: 5.1\% missing data on occupation, 3.4\% missing data on APOE \&4 carrier, $7.0 \%$ missing data on married, $0.34 \%$ missing data on leisure activities, $15 \%$ missing on data income, $6.3 \%$ missing data on antidepressant use, and $24 \%$ missing data on depressive symptoms.

The hazard ratios obtained in the sensitivity analysis (i.e., a worst-case scenario in which all those who were lost to followup due to death were assumed to have died with MCI) were comparable to those in the main analysis (Table 3). These results suggest a limited effect of informative censoring on the hazard ratios.

\section{Course of $\mathrm{MCl}$}

Of the 752 participants with incident MCI, 480 (64\%) had at least 1 follow-up visit after on average 2.4 years. Compared to the participants who had no follow-up after their first MCI diagnosis, those with follow-up were slightly younger (79.9 vs 81.4 years), were more likely to be part of the first 2 recruitment 


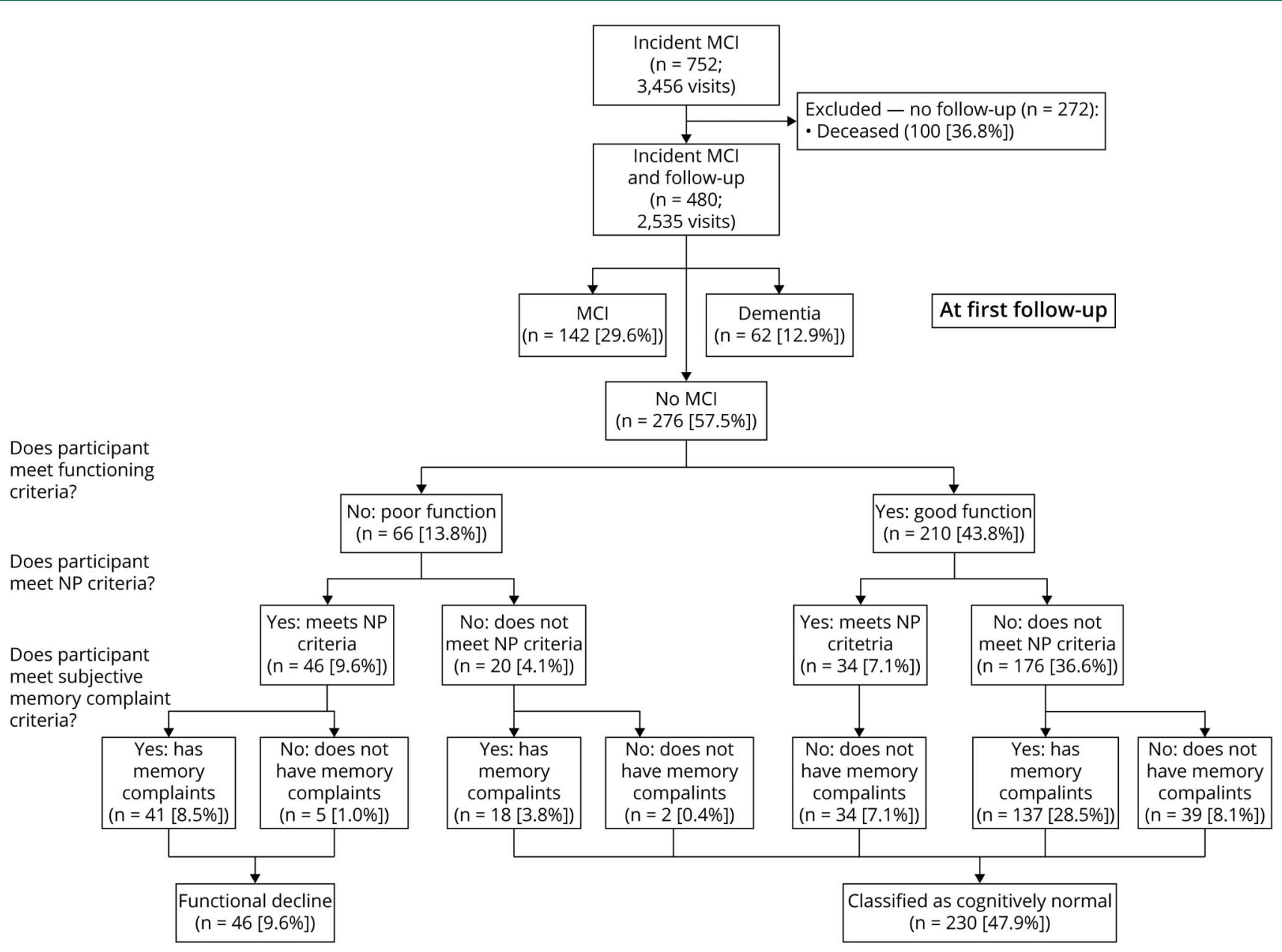

$\mathrm{MCl}=$ mild cognitive impairment

waves, had a lower medical burden (2.31 vs 2.63), reported more leisure activities (6.83 vs 6.19 ), and were more likely to have low levels of occupation and income.

Table 2 Incidence Rates by MCI Type ( $n=2,903 ; 13,449$ Person-Years)

\begin{tabular}{lll}
\hline MCI type & $\begin{array}{l}\text { No. of incident } \\
\text { MCl cases } \\
(\% \text { all MCl) }\end{array}$ & $\begin{array}{l}\text { Incidence rate per } \\
\mathbf{1 , 0 0 0} \text { person-y } \\
(\mathbf{9 5 \%} \mathrm{Cl})\end{array}$ \\
\hline Single-domain MCl amnestic & $224(30)$ & $17(15-19)$ \\
\hline Single-domain MCI nonamnestic & $338(45)$ & $25(23-28)$ \\
\hline Multidomain MCl amnestic & $117(16)$ & $8.6(7.3-10)$ \\
\hline Multidomain MCI nonamnestic & $73(9.7)$ & $5.4(4.3-6.9)$ \\
\hline All MCI & 752 & $56(52-60)$ \\
\hline $\begin{array}{l}\text { Dementia before MCI } \\
\text { All MCI and dementia } \\
\text { before MCl }\end{array}$ & 1,053 & $23(20-25)$ \\
\hline
\end{tabular}

Abbreviations: $\mathrm{Cl}=$ confidence interval; $\mathrm{MCl}=$ mild cognitive impairment.
Of the 480 incident MCI cases with at least 1 follow-up visit, $62(12.9 \%)$ progressed to dementia at follow-up, and 142 (29.6\%) continued to meet MCI criteria (i.e., sustained MCI diagnosis), namely presence of subjective memory complaint and objective cognitive impairment with preserved daily functioning and no diagnosis of dementia. The remaining 276 (57.5\%) participants did not meet full MCI criteria at their next follow-up visit. However, 66 of these 276 participants (24\%) had developed functional impairment that ruled them out of consideration for MCI, and 46 of those 66 in addition continued to have objective cognitive impairment. Due to functional impairment, these participants $(9.6 \%)$ did not meet the algorithmic criteria for MCI, but they did not meet the clinical criteria for dementia either. These participants were categorized as having functional decline. The remaining 230 participants (47.9\%) consisted of participants without dementia who had only subjective complaints, only objective cognitive impairment, only no functional impairment, or a combination of 2 of those 3 criteria but not all 3 criteria. Figure 2 provides a breakdown of the number of participants who did or did not meet the different criteria. 
Table 3 Results of Cox Regression Analyses of the Association of Predictors With Incident MCI

\begin{tabular}{|c|c|c|c|}
\hline & Separate models & Full model & Sensitivity analysis \\
\hline Characteristic & $\begin{array}{l}\text { Hazard ratio }(95 \% \mathrm{Cl}) \\
(\mathrm{n}=2,902,1,053 \text { events })\end{array}$ & $\begin{array}{l}\text { Hazard ratio }(95 \% \mathrm{Cl}) \\
(\mathrm{n}=2,902,1,053 \text { events })\end{array}$ & $\begin{array}{l}\text { Hazard ratio }(95 \% \mathrm{Cl}) \\
(\mathrm{n}=2,902,1,586 \text { events })\end{array}$ \\
\hline Age at baseline & - & $1.06(1.05-1.07), p<0.001$ & $1.06(1.05-1.07), p<0.001$ \\
\hline \multicolumn{4}{|l|}{ Cohort } \\
\hline 1992 & - & 1 (Reference) & 1 (Reference) \\
\hline 1999 & - & $0.93(0.80-1.09), p=0.372$ & $0.86(0.76-0.97), p=0.017$ \\
\hline 2009 & - & $0.68(0.56-0.84), p<0.001$ & $0.61(0.52-0.73), p<0.001$ \\
\hline Education & $0.93(0.92-0.94), p<0.001$ & $0.95(0.93-0.96), p<0.001$ & $0.96(0.95-0.98), p<0.001$ \\
\hline Women & $1.00(0.88-1.14), p=0.988$ & $0.88(0.77-1.02), p=0.097$ & $0.80(0.71-0.89), p<0.001$ \\
\hline Black & $0.94(0.82-1.07), p=0.336$ & $1.08(0.90-1.30), p=0.416$ & $1.09(0.94-1.25), p=0.235$ \\
\hline Hispanic & $1.50(1.33-1.70), p<0.001$ & $0.94(0.77-1.16), p=0.576$ & $0.84(0.72-0.99), p=0.037$ \\
\hline \multicolumn{4}{|l|}{ Occupation } \\
\hline Low & 1 (Reference) & 1 (Reference) & 1 (Reference) \\
\hline Medium & $0.67(0.57-0.77), p<0.001$ & $0.89(0.75-1.06), p=0.200$ & $0.96(0.83-1.10), p=0.511$ \\
\hline High & $0.50(0.43-0.60), p<0.001$ & $0.85(0.68-1.07), p=0.164$ & $0.93(0.78-1.11), p=0.394$ \\
\hline$A P O E \& 4$ carrier & $1.13(0.99-1.31), p=0.078$ & $1.18(1.02-1.36), p=0.025$ & $1.11(0.99-1.25), p=0.082$ \\
\hline Married & $0.92(0.80-1.06), p=0.234$ & $0.98(0.84-1.14), p=0.802$ & $0.99(0.87-1.12), p=0.868$ \\
\hline Medical burden & $1.09(1.04-1.14), p<0.001$ & $1.05(1.00-1.09), p=0.048$ & $1.08(1.04-1.12), p<0.001$ \\
\hline Leisure activities & $0.93(0.91-0.95), p<0.001$ & $0.98(0.95-1.00), p=0.072$ & $0.95(0.93-0.98), p<0.001$ \\
\hline \multicolumn{4}{|l|}{ Income } \\
\hline Low & 1 (Reference) & 1 (Reference) & 1 (Reference) \\
\hline Medium & $0.62(0.54-0.72), p<0.001$ & $0.80(0.68-0.95), p=0.009$ & $0.83(0.72-0.96), p=0.011$ \\
\hline High & $0.43(0.34-0.55), p<0.001$ & $0.73(0.54-1.00), p=0.053$ & $0.79(0.63-0.99), p=0.044$ \\
\hline Antidepressant use & $1.21(0.93-1.58), p=0.159$ & $1.15(0.87-1.51), p=0.329$ & $1.16(0.92-1.46), p=0.207$ \\
\hline Depressive symptoms & $1.19(0.99-1.43), p=0.069$ & $1.01(0.83-1.24), p=0.910$ & $1.05(0.89-1.24), p=0.570$ \\
\hline
\end{tabular}

Abbreviations: $\mathrm{Cl}=$ confidence interval; $\mathrm{MCl}=$ mild cognitive impairment.

Separate models include each predictor with adjustment for age and recruitment wave. Full models include all predictors and covariates (i.e., age and recruitment wave). The sensitivity analysis was performed on the full model; 1 case was dropped due to censoring before earliest event in stratum.

Table 4 shows the characteristics across the 4 outcome groups of MCI course: sustained MCI diagnosis, follow-up classification as cognitively normal, functional decline, and dementia. The functional decline group was more similar to the dementia group than the sustained MCI or cognitively normal groups.

Table 5 shows the relative risks of the associations of predictors with course of incident MCI across diagnostic groups compared with the group classified as cognitively normal at follow-up. In separate models per predictor adjusted for age and recruitment wave, having multidomain MCI was the only factor that reliably distinguished those who had a sustained MCI course from those who were classified as cognitively normal at follow-up. Having multidomain MCI, a higher medical burden, reduced leisure activities, antidepressant use, and depressive symptoms were associated with higher risk of functional decline group relative to classification as cognitively normal at follow-up. Apart from depressive symptoms, these associations remained in the full model. Higher risk of progression to dementia relative to classification as cognitively normal at follow-up was associated with having multidomain MCI, antidepressant use, and depressive symptoms in both separate models and the full model. In addition, higher risk of progression to dementia was associated with occupation and the presence of APOE $\varepsilon 4$ only in the full model. Years of education, sex/gender, race/ethnicity, marital status, and income were not associated with course of incident MCI. 
Table 4 Baseline Participant Characteristics Among Diagnostic Groups Based on MCI Status at First Follow-up After Incident $\mathrm{MCl}$ (Before Multiple Imputation) and Distribution of Variables Among Participants Without and With Missing Values

\begin{tabular}{|c|c|c|c|c|c|c|}
\hline \multirow[b]{2}{*}{ Characteristic } & \multicolumn{4}{|c|}{$\begin{array}{l}\text { Baseline participant characteristics among diagnostic groups } \\
\text { based on } \mathrm{MCl} \text { status }\end{array}$} & \multicolumn{2}{|c|}{$\begin{array}{l}\text { Distribution of variables among participants } \\
\text { without and with missing values }\end{array}$} \\
\hline & $\begin{array}{l}\text { Cognitively } \\
\text { normal }\end{array}$ & Sustained $\mathrm{MCl}$ & Dementia & $\begin{array}{l}\text { Functional } \\
\text { decline }\end{array}$ & $\begin{array}{l}\text { Data } \\
\text { complete }\end{array}$ & $\begin{array}{l}\text { Data } \\
\text { incomplete }\end{array}$ \\
\hline No. (\%) & $230(47.9)$ & $142(29.6)$ & $62(12.9)$ & $46(9.6)$ & $263(49 \%)$ & $244(51 \%)$ \\
\hline Age, mean (SD), y & $78.5(5.5)$ & $79.5(6.0)$ & $83.6(5.7)$ & $83.1(6.3)$ & $79.8(6.2)$ & $80.0(5.9)$ \\
\hline Education mean (SD), y & $9.92(4.8)$ & $9.71(4.8)$ & $9.11(5.2)$ & $9.30(4.7)$ & $9.78(4.8)$ & $9.61(4.9)$ \\
\hline Follow-up, mean (SD), mo & $28.2(10.5)$ & $28.6(10.6)$ & $33.2(10.1)$ & $30.0(11.5)$ & $31.2(11.4)$ & $27.2(9.6)$ \\
\hline \multicolumn{7}{|l|}{ Cohort, n (\%) } \\
\hline 1992 & $86(37.4)$ & $59(41.5)$ & $25(40.3)$ & $19(41.3)$ & $31(13.1)$ & $158(64.8)$ \\
\hline 1999 & $108(47.0)$ & $68(47.9)$ & $28(45.2)$ & $22(47.8)$ & $154(65.3)$ & $72(29.5)$ \\
\hline 2009 & $36(15.7)$ & $15(10.6)$ & $9(14.5)$ & $5(10.9)$ & $51(21.6)$ & $14(5.7)$ \\
\hline Women, n (\%) & $163(70.9)$ & $102(71.8)$ & $42(67.7)$ & $36(78.3)$ & $164(69.5)$ & $179(73.4)$ \\
\hline Black, n (\%) & $67(29.1)$ & $51(35.9)$ & $22(35.5)$ & $12(26.1)$ & $68(28.8)$ & $84(34.4)$ \\
\hline Hispanic, n (\%) & $106(46.1)$ & $58(40.8)$ & $29(46.8)$ & $21(45.7)$ & $113(47.9)$ & $101(41.4)$ \\
\hline \multicolumn{7}{|l|}{ Occupation, n (\%) } \\
\hline Low & $130(58.3)$ & $82(61.2)$ & $35(59.3)$ & $28(63.6)$ & $137(58.1)$ & $138(61.6)$ \\
\hline Medium & $53(23.8)$ & $29(21.6)$ & $16(27.1)$ & $11(25.0)$ & $52(22.0)$ & $57(25.4)$ \\
\hline High & $40(17.9)$ & $23(17.2)$ & $8(13.6)$ & $5(11.4)$ & 47 (19.9) & $29(12.9)$ \\
\hline APOE \&4 carrier, $\mathrm{n}(\%)$ & $60(26.2)$ & $36(26.5)$ & $25(41.7)$ & $13(28.3)$ & $65(27.5)$ & $69(29.4)$ \\
\hline Married, n (\%) & $56(32.4)$ & $25(25.0)$ & $12(26.1)$ & $9(26.5)$ & $68(28.8)$ & $34(29.1)$ \\
\hline Medical burden, mean (SD) & $2.33(1.4)$ & $2.25(1.5)$ & $2.13(1.4)$ & $2.67(1.4)$ & $2.67(1.4)$ & $1.97(1.4)$ \\
\hline Leisure activities, mean (SD) & $6.95(2.3)$ & $7.25(2.2)$ & $6.16(2.3)$ & $5.83(2.0)$ & $6.79(2.3)$ & $6.86(2.4)$ \\
\hline \multicolumn{7}{|l|}{ Income, n (\%) } \\
\hline Low & $82(44.6)$ & $54(45.4)$ & $25(52.1)$ & $15(44.1)$ & $83(35.2)$ & $93(62.4)$ \\
\hline Medium & $89(48.4)$ & $58(48.7)$ & 19 (39.6) & $18(52.9)$ & $132(55.9)$ & $52(34.9)$ \\
\hline High & $13(7.1)$ & $7(5.9)$ & $4(8.3)$ & $1(2.9)$ & $21(8.9)$ & $4(2.7)$ \\
\hline Antidepressant use, $\mathbf{n}(\%)$ & $12(5.9)$ & $8(6.5)$ & $10(17.5)$ & $8(18.2)$ & $23(9.7)$ & $15(7.9)$ \\
\hline Depressive symptoms, $n$ (\%) & $17(10.3)$ & $12(12.4)$ & $9(20.9)$ & $10(29.4)$ & $34(14.4)$ & $14(13.6)$ \\
\hline Multidomain $\mathrm{MCl}, \mathrm{n}(\%)$ & $40(17.4)$ & $42(29.6)$ & $25(40.3)$ & $16(34.8)$ & $50(21.2)$ & $73(29.9)$ \\
\hline
\end{tabular}

Abbreviation: $\mathrm{MCl}=$ mild cognitive impairment.

Participant characteristics based on data before imputation: 4.1\% missing data on occupation; $1.8 \%$ missing data on APOE $\varepsilon 4$ carrier; $26 \%$ missing data on married; $20 \%$ missing data on income; $11 \%$ missing data on antidepressant use; $30 \%$ missing data on depressive symptoms.

\section{Discussion}

We examined predictors of incident MCI and predictors of diagnostic status at the next follow-up visit after incident $\mathrm{MCI}$ in a large racially and ethnically diverse community-based study. We found that having an APOE $\& 4$ allele and higher medical burden increased the risk of incident MCI, whereas more years of education, more leisure activities, and higher income decreased this risk. In the incident $\mathrm{MCI}$ group, antidepressant use, depressive symptoms, and multidomain MCI were associated with a worse follow-up diagnosis (i.e., functional decline or dementia) relative to the group who fell within cognitively normal limits at followup. While higher medical burden and reduced leisure activities were in addition associated specifically with a higher risk of functional decline, the presence of $A P O E \& 4$ was specifically associated with progression from MCI to dementia. 
Table 5 Relative Risks of Belonging to the Sustained MCl, Functional Decline, and Dementia Groups Compared to the Group Classified as Cognitively Normal at Follow-up, Computed With Poisson Regressions Corrected for Age and Time of Follow-up

\begin{tabular}{|c|c|c|c|c|c|c|}
\hline & \multicolumn{2}{|c|}{$\begin{array}{l}\text { Sustained } \mathrm{MCl} \text { vs cognitively normal } \\
\operatorname{RR}(95 \% \mathrm{CI})(\mathrm{N}=372[142,230])\end{array}$} & \multicolumn{2}{|c|}{$\begin{array}{l}\text { Functional decline vs cognitively } \\
\text { normal } R R(95 \% \mathrm{CI})(\mathrm{N}=276[46,230])\end{array}$} & \multicolumn{2}{|c|}{$\begin{array}{l}\text { Dementia vs cognitively normal } \\
\operatorname{RR}(95 \% \mathrm{CI})(\mathrm{N}=292[62,230]\end{array}$} \\
\hline & Separate models & Full model & Separate models & Full model & Separate models & Full model \\
\hline Age at incidence & - & $\begin{array}{l}1.02(1.00-1.04) \\
p=0.073\end{array}$ & - & $\begin{array}{l}1.10(1.05-1.14) \\
p<0.001\end{array}$ & - & $\begin{array}{l}1.10(1.07-1.14) \\
p<0.001\end{array}$ \\
\hline \multicolumn{7}{|l|}{ Cohort } \\
\hline 1992 & - & 1 (Reference) & - & 1 (Reference) & - & 1 (Reference) \\
\hline 1999 & - & $\begin{array}{l}0.80(0.58-1.10) \\
p=0.170\end{array}$ & - & $\begin{array}{l}0.74(0.38-1.45) \\
p=0.3830\end{array}$ & - & $\begin{array}{l}1.00(0.59-1.67) \\
p=0.990\end{array}$ \\
\hline 2009 & - & $\begin{array}{l}0.86(0.52-1.43) \\
p=0.560\end{array}$ & - & $\begin{array}{l}0.57(0.21-1.54) \\
p=0.269\end{array}$ & - & $\begin{array}{l}1.22(0.66-2.26) \\
p=0.527\end{array}$ \\
\hline Education & $\begin{array}{l}0.99(0.96-1.02) \\
p=0.460\end{array}$ & $\begin{array}{l}0.99(0.95-1.03) \\
p=0.623\end{array}$ & $\begin{array}{l}0.98(0.93-1.03) \\
p=0.416\end{array}$ & $\begin{array}{l}0.99(0.91-1.07) \\
p=0.765\end{array}$ & $\begin{array}{l}0.97(0.92-1.01) \\
p=0.129\end{array}$ & $\begin{array}{l}0.96(0.90-1.02) \\
p=0.142\end{array}$ \\
\hline Women & $\begin{array}{l}1.05(0.77-1.42) \\
p=0.767\end{array}$ & $\begin{array}{l}0.94(0.68-1.30) \\
p=0.697\end{array}$ & $\begin{array}{l}1.29(0.72-2.33) \\
p=0.390\end{array}$ & $\begin{array}{l}1.19(0.63-2.23) \\
p=0.597\end{array}$ & $\begin{array}{l}0.80(0.53-1.22) \\
p=0.309\end{array}$ & $\begin{array}{l}0.71(0.44-1.15) \\
p=0.169\end{array}$ \\
\hline Black & $\begin{array}{l}1.25(0.95-1.65) \\
p=0.108\end{array}$ & $\begin{array}{l}1.28(0.88-1.86) \\
p=0.199\end{array}$ & $\begin{array}{l}1.07(0.59-1.95) \\
p=0.822\end{array}$ & $\begin{array}{l}1.69(0.77-3.72) \\
p=0.193\end{array}$ & $\begin{array}{l}1.16(0.77-1.77) \\
p=0.473\end{array}$ & $\begin{array}{l}1.89(0.99-3.61) \\
p=0.054\end{array}$ \\
\hline Hispanic & $\begin{array}{l}0.96(0.73-1.27) \\
p=0.770\end{array}$ & $\begin{array}{l}1.00(0.62-1.63) \\
p=0.987\end{array}$ & $\begin{array}{l}1.14(0.68-1.90) \\
p=0.611\end{array}$ & $\begin{array}{l}1.55(0.66-3.65) \\
p=0.311\end{array}$ & $\begin{array}{l}1.34(0.86-2.08) \\
p=0.195\end{array}$ & $\begin{array}{l}1.63(0.80-3.34) \\
p=0.178\end{array}$ \\
\hline $\begin{array}{l}\text { Occupation, } \\
\text { medium-high }\end{array}$ & $\begin{array}{l}0.90(0.68-1.19) \\
p=0.450\end{array}$ & $\begin{array}{l}0.96(0.68-1.37) \\
p=0.829\end{array}$ & $\begin{array}{l}0.91(0.53-1.56) \\
p=0.738\end{array}$ & $\begin{array}{l}1.23(0.61-2.47) \\
p=0.558\end{array}$ & $\begin{array}{l}1.06(0.68-1.64) \\
p=0.806\end{array}$ & $\begin{array}{l}1.81(1.07-3.06) \\
p=0.026\end{array}$ \\
\hline APOE $\varepsilon 4$ carrier & $\begin{array}{l}1.08(0.79-1.48) \\
p=0.641\end{array}$ & $\begin{array}{l}1.04(0.76-1.42) \\
p=0.791\end{array}$ & $\begin{array}{l}1.21(0.68-2.15) \\
p=0.522\end{array}$ & $\begin{array}{l}1.13(0.63-2.03) \\
p=0.691\end{array}$ & $\begin{array}{l}1.47(0.98-2.18) \\
p=0.061\end{array}$ & $\begin{array}{l}1.65(1.02-2.65), \\
p=0.040\end{array}$ \\
\hline Married & $\begin{array}{l}0.90(0.63-1.29) \\
p=0.569\end{array}$ & $\begin{array}{l}0.90(0.61-1.32) \\
p=0.584\end{array}$ & $\begin{array}{l}0.86(0.45-1.65) \\
p=0.651\end{array}$ & $\begin{array}{l}0.85(0.43-1.72) \\
p=0.658\end{array}$ & $\begin{array}{l}0.98(0.60-1.61) \\
p=0.944\end{array}$ & $\begin{array}{l}0.90(0.53-1.56) \\
p=0.716\end{array}$ \\
\hline Medical burden & $\begin{array}{l}1.00(0.91-1.11) \\
p=0.933\end{array}$ & $\begin{array}{l}1.01(0.91-1.12) \\
p=0.847\end{array}$ & $\begin{array}{l}1.17(0.98-1.39) \\
p=0.075\end{array}$ & $\begin{array}{l}1.21(0.99-1.48) \\
p=0.057\end{array}$ & $\begin{array}{l}0.96(0.83-1.11) \\
p=0.562\end{array}$ & $\begin{array}{l}0.90(0.77-1.07) \\
p=0.231\end{array}$ \\
\hline Leisure activity & $\begin{array}{l}1.01(0.96-1.08) \\
p=0.651\end{array}$ & $\begin{array}{l}1.02(0.96-1.08) \\
p=0.460\end{array}$ & $\begin{array}{l}0.86(0.77-0.96) \\
p=0.010\end{array}$ & $\begin{array}{l}0.89(0.79-1.00) \\
p=0.042\end{array}$ & $\begin{array}{l}0.94(0.85-1.03) \\
p=0.183\end{array}$ & $\begin{array}{l}0.99(0.91-1.09) \\
p=0.892\end{array}$ \\
\hline Income, medium-high & $\begin{array}{l}0.89(0.65-1.22) \\
p=0.475\end{array}$ & $\begin{array}{l}0.87(0.60-1.26) \\
p=0.468\end{array}$ & $\begin{array}{l}0.90(0.5-1.65) \\
p=0.742\end{array}$ & $\begin{array}{l}1.02(0.51-2.03) \\
p=0.967\end{array}$ & $\begin{array}{l}0.74(0.46-1.19) \\
p=0.213\end{array}$ & $\begin{array}{l}0.92(0.55-1.55) \\
p=0.761\end{array}$ \\
\hline Antidepressant use & $\begin{array}{l}1.18(0.68-2.04) \\
p=0.558\end{array}$ & $\begin{array}{l}1.32(0.74-2.36) \\
p=0.348\end{array}$ & $\begin{array}{l}2.71(1.44-5.12) \\
p=0.002\end{array}$ & $\begin{array}{l}2.27(1.11-4.61) \\
p=0.024\end{array}$ & $\begin{array}{l}1.90(1.24-2.91) \\
p=0.003\end{array}$ & $\begin{array}{l}2.43(1.47-4.02) \\
p=0.001\end{array}$ \\
\hline Depressive symptoms & $\begin{array}{l}1.02(0.63-1.65) \\
p=0.929\end{array}$ & $\begin{array}{l}1.04(0.64-1.69) \\
p=0.883\end{array}$ & $\begin{array}{l}2.10(1.09-4.04) \\
p=0.027\end{array}$ & $\begin{array}{l}1.67(0.81-3.42) \\
p=0.160\end{array}$ & $\begin{array}{l}1.84(1.03-3.28) \\
p=0.038\end{array}$ & $\begin{array}{l}1.80(1.06-3.06) \\
p=0.030\end{array}$ \\
\hline Multidomain $\mathrm{MCl}$ & $\begin{array}{l}1.52(1.16-1.99) \\
p=0.003\end{array}$ & $\begin{array}{l}1.53(1.16-2.00) \\
p=0.002\end{array}$ & $\begin{array}{l}2.03(1.20-3.43) \\
p=0.009\end{array}$ & $\begin{array}{l}1.98(1.17-3.35) \\
p=0.011\end{array}$ & $\begin{array}{l}2.07(1.34-3.19) \\
p=0.001\end{array}$ & $\begin{array}{l}2.11(1.31-3.39) \\
p=0.002\end{array}$ \\
\hline
\end{tabular}

Abbreviation: $\mathrm{Cl}$ = confidence interval; $\mathrm{MCl}=$ mild cognitive impairment; $\mathrm{RR}=$ relative risk.

Separate models include each predictor with adjustment for age and recruitment wave. Full models include all predictors and covariates (i.e., age and recruitment wave). $\mathrm{N}$ indicates the total number of participants with number of participants per category in brackets.

Years of schooling has consistently been shown to predict incident $\mathrm{MCI}$ in previous studies, but the relation of income or occupation to incident $\mathrm{MCI}$ is not well understood. ${ }^{31}$ In separate models, we observed that all 3 factors, that is, more years of education, higher income, and higher occupational level, were associated with lower risk of incident MCI. All 3 predictors are highly related to each other (e.g., household income is heavily determined by educational attainment and occupation), and in a full model, occupation dropped out as a predictor of incident MCI, while household income and years of education remained. Income is more proximal in time to cognitive aging than education and may be a relevant reflection of socioeconomic status among retirees. In this group, a higher income has been associated with better access to health care, increased social activities, fewer daily stressors, and slower cognitive decline. ${ }^{32}$ 
On the basis of studies that show that the incidence of dementia is higher among non-Hispanic Black and Hispanic older adults compared to White individuals, we also expected higher MCI incidence in these groups. ${ }^{33}$ We observed that in separate models, Hispanics were at higher risk of incident MCI, but this association did not survive the fully adjusted model. This change is likely due to collinearity of this group membership with other predictors; for example, the Hispanic population sampled in the WHICAP cohort, representative of the Northern Manhattan community, is on average lower educated. ${ }^{34}$ Future research is warranted to fully deconstruct differences across race and ethnicity with regard to risk and associated predictors of incident MCI, diagnostic status after incident MCI, and the longitudinal course between incident MCI and a diagnosis of dementia.

Our observation that a high proportion of people with incident MCI no longer met full MCI criteria at follow-up and fell within the cognitively normal classification again may be explained by several factors. First, the percentage of diagnostic reversion in community-based studies is higher than in clinicbased studies, which often report a lower percentage of reversion. $^{8,10,13}$ In most clinic-based studies, follow-up diagnosis is assigned without blinding the clinician to earlier diagnoses, while clinicians may be more likely to give a diagnosis of MCI if it has been given before. ' Furthermore, clinic-based studies have a higher base rate of people who progress to dementia than population-based studies. ${ }^{35} \mathrm{~A}$ lower, but still considerable, reversion rate of $25 \%$ was found in those with incident MCI in a clinic-based sample. ${ }^{12}$ Fiftyone percent of the participants had multidomain MCI in this clinic-based sample, while we reported only $26 \%$ of those with incident MCI to be characterized as having multidomain MCI. In addition, the mean time to follow-up was shorter in our study compared to that of Michaud et al. (2.4 vs 4.3 years). A shorter follow-up time is likely related to less advanced disease at follow-up; slight fluctuations above and below the cognitive test cutoffs may be more likely earlier in the disease process. In our study, a large number of individuals with incident MCI did not meet full MCI criteria anymore because their performance on objective cognitive tests was above the 1.5 SD cutoffs at follow-up.

We found that participating in fewer leisure activities at the MCI incidence visit was associated with higher likelihood of being classified as having functional decline at follow-up compared to those whose cognitive test scores improved. This finding suggests that a decline in daily functioning is preceded by reduced participation in leisure activities. The functional decline group comprised participants who no longer fulfilled MCI criteria because their daily function declined, but their neuropsychological test scores remained mildly impaired, not yet meeting dementia criteria at consensus conference. In other words, these individuals fall in between the MCI and dementia classifications. We found multiple similarities between the functional decline group and the group who progressed to dementia. Although other population-based cohorts specify a maximum functional impairment allowed for the diagnosis of MCI, ${ }^{8,9}$ these studies have not described this functional decline subgroup. The identification of this group is a result of diagnostic procedures in this study (and possibly other studies), and we do not posit this group as a new nosologic entity. However, it is important to understand the trajectory of this group as being distinct from that of those who no longer meet MCI criteria for other reasons (e.g., improved cognitive function) in future population-based cohort studies because of their similarity to those who progress to dementia.

The presence of depressive symptoms and antidepressant use were associated with higher incident MCI risk in the model adjusted only for age and recruitment cohort, but this association did not survive in the fully adjusted model. Our prior study reported no association between baseline depression and incident $\mathrm{MCI}{ }^{36}$ while other studies did observe a relationship. ${ }^{37}$ Nonetheless, depressive symptoms and antidepressant use were strongly associated with progression to the functional decline group or dementia at follow-up in our participants with incident MCI, not with classification as cognitively normal. The observation that depressive symptoms at the time of neuropsychological assessment do not predict classification as cognitively normal at follow-up is of particular relevance because, for example, motivational symptoms of depression can temporarily affect attentional processes and performance on memory tests. ${ }^{38,39}$ In addition, the association between depressive symptoms and antidepressant use and the predementia and dementia groups strengthens the previously reported evidence for depressive symptoms as a predictor of progression to dementia. ${ }^{40,41}$

Strengths of this study include a large sample with a relatively high proportion of Black and Hispanic participants and extensive follow-up, which allowed the study of outcomes after observation of incident MCI, which is likely to lead to less biased estimates than follow-up of prevalent MCI cases. Black and Hispanic older adults are less likely to have a formal diagnosis of dementia, and when they are diagnosed, their disease is more severe. ${ }^{42}$ This means that clinic-based cohorts are not appropriate for research on progression of cognitive impairment across different races/ethnicities. ${ }^{43}$ The WHICAP cohort provides an opportunity to study the course of incident $\mathrm{MCI}$ in a representative cohort and to do so among Black and Hispanic people without the kind of enrollment bias that affects clinic-based studies such as National $\mathrm{Alz}$ heimer's Coordinating Center.

Although this extensive cohort allowed study of follow-up after incident MCI, the proportion with follow-up after incident MCI is relatively small, and the follow-up time after incident MCI is relatively short. Future research should investigate the course of MCI across multiple follow-up visits across a longer period of time to further dissect which factors are most informatively related to development of 
neurodegenerative disease. Another potential limitation is that we do not yet have plasma Alzheimer disease biomarker information on our participants, and thus, this information could not take these disease markers into account.

This community-based study including a relatively high proportion of Black and Hispanic participants showed that a large proportion of individuals with incident MCI may fall within cognitively normal limits at follow-up. Predictors of incident MCI differed demonstrably from those of subsequent MCI course; these findings can refine expectations for cognitive and functional course of those presenting with MCI.

\section{Acknowledgment}

This manuscript has been reviewed by WHICAP investigators for scientific content and consistency of data interpretation with previous WHICAP Study publications. The authors acknowledge the WHICAP study participants and the WHICAP research and support staff for their contributions to this study.

\section{Study Funding}

Data collection and sharing for this project were supported by the WHICAP (PO1AG07232, R01AG037212, RF1AG054023) funded by the National Institute on Aging. This publication was supported by the National Center for Advancing Translational Sciences, NIH, through grant UL1TR001873. Mirjam I. Geerlings was funded by a grant of Alzheimer Nederland (grant WE03-2017-07).

\section{Disclosure}

The authors report no disclosures relevant to the manuscript. Go to Neurology.org/N for full disclosures.

\section{Publication History}

Received by Neurology November 19, 2020. Accepted in final form September 29, 2021.

Appendix Authors

\begin{tabular}{|c|c|c|}
\hline Name & Location & Contribution \\
\hline $\begin{array}{l}\text { Milou J. } \\
\text { Angevaare, } \\
\text { MD }\end{array}$ & $\begin{array}{l}\text { Department of Neurology } \\
\text { and Taub Institute for } \\
\text { Research on Alzheimer's } \\
\text { Disease and The Aging Brain, } \\
\text { College of Physicians and } \\
\text { Surgeons, Columbia } \\
\text { University, New York, NY; } \\
\text { Julius Center for Health } \\
\text { Sciences and Primary Care, } \\
\text { University Medical Center } \\
\text { Utrecht, the Netherlands; } \\
\text { Amsterdam UMC, Vrije } \\
\text { Universiteit Amsterdam, } \\
\text { Department of Medicine for } \\
\text { Older People, Amsterdam } \\
\text { Public Health Research } \\
\text { Institute, the Netherlands }\end{array}$ & $\begin{array}{l}\text { Design of the study, analysis } \\
\text { and interpretation of the } \\
\text { data, drafting and revising } \\
\text { the manuscript }\end{array}$ \\
\hline
\end{tabular}

Appendix (continued)

\begin{tabular}{lll}
\hline Name & Location & Contribution \\
\hline Jet M.J. & $\begin{array}{l}\text { Department of Neurology } \\
\text { Vonk, PhD Taub Institute for } \\
\text { Research on Alzheimer's } \\
\text { Disease and The Aging Brain, } \\
\text { College of Physicians and } \\
\text { Surgeons, Columbia }\end{array}$ & $\begin{array}{l}\text { Analysis and interpretation } \\
\text { of the data, revising the } \\
\text { manuscript }\end{array}$ \\
& University, New York, NY; \\
& Julius Center for Health \\
& Sciences and Primary Care, \\
& University Medical Center \\
& Utrecht, the Netherlands
\end{tabular}

\begin{tabular}{|c|c|c|}
\hline $\begin{array}{l}\text { Laiss } \\
\text { Bertola, } \\
\text { PhD }\end{array}$ & $\begin{array}{l}\text { Department of Neurology and } \\
\text { Taub Institute for Research on } \\
\text { Alzheimer's Disease and The } \\
\text { Aging Brain, College of } \\
\text { Physicians and Surgeons, } \\
\text { Columbia University, New } \\
\text { York, NY; National Institute of } \\
\text { Science and Technology in } \\
\text { Molecular Medicine, Federal } \\
\text { University of Minas Gerais, } \\
\text { Belo Horizonte, Brazil }\end{array}$ & $\begin{array}{l}\text { Design of the study, analysis } \\
\text { and interpretation of the } \\
\text { data, revising the manuscript }\end{array}$ \\
\hline $\begin{array}{l}\text { Laura } \\
\text { Zahodne, } \\
\text { PhD }\end{array}$ & $\begin{array}{l}\text { Department of Neurology } \\
\text { and Taub Institute for } \\
\text { Research on Alzheimer's } \\
\text { Disease and The Aging Brain, } \\
\text { College of Physicians and } \\
\text { Surgeons, Columbia } \\
\text { University, New York, NY }\end{array}$ & $\begin{array}{l}\text { Analysis and interpretation } \\
\text { of the data, revising the } \\
\text { manuscript }\end{array}$ \\
\hline
\end{tabular}

Caitlin Wei- Department of Neurology Ming and Taub Institute for

Watson, Research on Alzheimer's

MSc Disease and The Aging Brain,

Analysis and interpretation of the data, revising the College of Physicians and Surgeons, Columbia University, New York, NY

\begin{tabular}{|c|c|c|}
\hline $\begin{array}{l}\text { Amelia } \\
\text { Boehme, } \\
\text { PhD }\end{array}$ & $\begin{array}{l}\text { Department of Neurology } \\
\text { and Taub Institute for } \\
\text { Research on Alzheimer's } \\
\text { Disease and The Aging Brain, } \\
\text { College of Physicians and } \\
\text { Surgeons, Columbia } \\
\text { University, New York, NY }\end{array}$ & $\begin{array}{l}\text { Analysis and interpretation } \\
\text { of the data, revising the } \\
\text { manuscript }\end{array}$ \\
\hline $\begin{array}{l}\text { Nicole } \\
\text { Schupf, } \\
\text { PhD }\end{array}$ & $\begin{array}{l}\text { Department of Neurology } \\
\text { and Taub Institute for } \\
\text { Research on Alzheimer's } \\
\text { Disease and The Aging Brain, } \\
\text { College of Physicians and } \\
\text { Surgeons, Columbia } \\
\text { University, New York, NY }\end{array}$ & $\begin{array}{l}\text { Analysis and interpretation } \\
\text { of the data, revising the } \\
\text { manuscript }\end{array}$ \\
\hline
\end{tabular}

\begin{tabular}{ll}
\hline Richard & Department of Neurology \\
Mayeux, & and Taub Institute for \\
MD & Research on Alzheimer's \\
& Disease and The Aging Brain, \\
& College of Physicians and \\
& Surgeons, Columbia \\
& University, New York, NY
\end{tabular}

\begin{tabular}{lll}
\hline $\begin{array}{l}\text { Mirjam I. } \\
\text { Geerlings, } \\
\text { PhD }\end{array}$ & $\begin{array}{l}\text { Julius Center for Health } \\
\text { Sciences and Primary Care, } \\
\text { University Medical Center } \\
\text { Utrecht, the Netherlands }\end{array}$ & $\begin{array}{l}\text { Analysis and interpretation } \\
\text { of the data, revising the } \\
\text { manuscript }\end{array}$ \\
\hline $\begin{array}{l}\text { Jennifer J. } \\
\text { Manly, PhD }\end{array}$ & $\begin{array}{l}\text { Department of Neurology } \\
\text { and Taub Institute for } \\
\text { Research on Alzheimer's } \\
\text { Disease and The Aging Brain, } \\
\text { College of Physicians and } \\
\text { Surgeons, Columbia } \\
\text { University, New York, NY }\end{array}$ & $\begin{array}{l}\text { Design of the study, analysis } \\
\text { and interpretation of the } \\
\text { data, drafting and revising } \\
\text { the manuscript }\end{array}$ \\
\end{tabular}




\section{References}

1. Petersen RC, Smith GE, Waring SC, Ivnik RJ, Tangalos EG, Kokmen E. Mild cognitive impairment: clinical characterization and outcome. Arch Neurol. 1999;56(3): 303-308.

2. Petersen RC, Roberts RO, Knopman DS, et al. Mild cognitive impairment: ten years later. Arch Neurol. 2009;66(12):1447-1455.

3. Solfrizzi V, Panza F, Colacicco AM, et al. Vascular risk factors, incidence of MCI, and rates of progression to dementia. Neurology. 2004;63(12):1882-1891.

4. Manly JJ, Tang MX, Schupf N, Stern Y, Vonsattel JP, Mayeux R. Frequency and course of mild cognitive impairment in a multiethnic community. Ann Neurol. 2008; 63(4):494-506

5. Tervo $S$, Kivipelto $M$, Hänninen $T$, et al. Incidence and risk factors for mild cognitive impairment: a population-based three-year follow-up study of cognitively healthy elderly subjects. Dement Geriatr Cogn Disord. 2004;17(3):196-203.

6. Caracciolo B, Palmer K, Monastero R, Winblad B, Bäckman L, Fratiglioni L. Occurrence of cognitive impairment and dementia in the community: a 9-year-long prospective study. Neurology. 2008;70(19 pt 2):1778-1785.

7. Luck T, Riedel-Heller S, Luppa M, et al. Risk factors for incident mild cognitive impairment: results from the German Study on Ageing, Cognition and Dementia in Primary Care Patients (AgeCoDe). Acta Psychiatrica Scand. 2010;121(4):260-272.

8. Ganguli M, Snitz BE, Saxton JA, et al. Outcomes of mild cognitive impairment by definition: a population study. Arch Neurol. 2011;68(6):761-767.

9. Sachdev PS, Lipnicki DM, Crawford J, et al. Factors predicting reversion from mild cognitive impairment to normal cognitive functioning: a population-based study. PLoS one. 2013;8(3):e59649.

10. Koepsell TD, Monsell SE. Reversion from mild cognitive impairment to normal or nearnormal cognition: risk factors and prognosis. Neurology. 2012;79(15):1591-1598.

11. Nordlund A, Rolstad S, Klang O, Edman A, Hansen S, Wallin A. Two-year outcome of MCI subtypes and aetiologies in the Göteborg MCI study. J Neurol Neurosurg Psychiatry. 2010;81(5):541-546.

12. Michaud TL, Su D, Siahpush M, Murman DL. The risk of incident mild cognitive impairment and progression to dementia considering mild cognitive impairment subtypes. Dement Geriatr Cogn Dis Extra. 2017;7(1):15-29.

13. Canevelli M, Grande G, Lacorte E, et al. Spontaneous reversion of mild cognitive impairment to normal cognition: a systematic review of literature and meta-analysis. J Am Med Dir Assoc. 2016;17(10):943-948

14. Pandya SY, Clem MA, Silva LM, Woon FL. Does mild cognitive impairment always lead to dementia? A review. J Neurol Sci. 2016;369:57-62.

15. Allaire JC, Gamaldo A, Ayotte BJ, Sims R, Whitfield K. Mild cognitive impairment and objective instrumental everyday functioning: the Everyday Cognition Battery Memory Test. J Am Geriatr Soc. 2009;57(1):120-125.

16. Aretouli E, Brandt J. Everyday functioning in mild cognitive impairment and its relationship with executive cognition. Int J Geriatr Psychiatry. 2010;25(3):224-233.

17. Luchsinger JA, Tang MX, Stern Y, Shea S, Mayeux R. Diabetes mellitus and risk of Alzheimer's disease and dementia with stroke in a multiethnic cohort. Am J Epidemiol. 2001;154(7):635-641.

18. Manly JJ, Bell-McGinty S, Tang MX, Schupf N, Stern Y, Mayeux R. Implementing diagnostic criteria and estimating frequency of mild cognitive impairment in an urban community. Arch Neurol. 2005;62(11):1739-1746.

19. Tang MX, Cross $\mathrm{P}$, Andrews $\mathrm{H}$, et al. Incidence of $\mathrm{AD}$ in African-Americans, Caribbean Hispanics, and Caucasians in Northern Manhattan. Neurology. 2001;56(1): 49-56.

20. Gurland B, Kuriansky J, Sharpe L, Simon R, Stiller P, Birkett P. The Comprehensive Assessment and Referral Evaluation (CARE): rationale, development and reliability. Int J Aging Hum Dev. 1978;8(1):9-42.

21. American Psychiatric Association. Diagnostic and Statistical Manual of Mental Disorders, 3rd Revised ed. American Psychiatric Association; 1987.
22. United States Office of Management and Budget. Revisions to the Standards for the Classification of Federal Data on Race and Ethnicity (October 30, 1997). Standards for Maintaining, Collecting, and Presenting Federal Data on Race and Ethnicity. Washington, DC: United States Office of Management and Budget, 1997.

23. Avila JF, Vonk JMJ, Verney SP, et al. Sex/gender differences in cognitive trajectories vary as a function of race/ethnicity. Alzheimers Dement. 2019;15(12):1516-1523.

24. Scarmeas N, Levy G, Tang MX, Manly J, Stern Y. Influence of leisure activity on the incidence of Alzheimer's disease. Neurology. 2001;57(12):2236-2242.

25. Zahodne LB, Manly JJ, MacKay-Brandt A, Stern Y. Cognitive declines precede and predict functional declines in aging and Alzheimer's disease. PLoS One. 2013;8: e73645.

26. Mayeux R, Ottman R, Maestre G, et al. Synergistic effects of traumatic head injury and apolipoprotein-epsilon 4 in patients with Alzheimer's disease. Neurology. 1995;45(3 pt 1):555-557.

27. Irwin M, Artin KH, Oxman MN. Screening for depression in the older adult: criterion validity of the 10-item Center for Epidemiological Studies Depression Scale (CES-D). Arch Intern Med. 1999;159(15):1701-1704.

28. Kleinbaum D, Klein M. Survival Analysis: A Self-Learning Text. Springer-Verlag; 2012.

29. Zou G. A modified Poisson regression approach to prospective studies with binary data. Am J Epidemiol. 2004;159(7):702-706.

30. IBM Corp. IBM SPSS Statistics for Windows, Version 25, IBM SPSS Corp; 2017.

31. Luck T, Luppa M, Briel S, Riedel-Heller SG. Incidence of mild cognitive impairment: a systematic review. Dement Geriatr Cogn Disord. 2010;29(2):164-175.

32. Zahodne LB, Stern Y, Manly JJ. Differing effects of education on cognitive decline in diverse elders with low versus high educational attainment. Neuropsychology. 2015; 29(4):649-657.

33. Anderson NB, Bulatao RA, Cohen B, on Race P, Council NR. Ethnic Differences in Dementia and Alzheimer's Disease: Critical Perspectives on Racial and Ethnic Differences in Health in Late Life. National Academies Press; 2004.

34. Vonk JMJ, Arce Rentería M, Avila JF, et al. Secular trends in cognitive trajectories of diverse older adults. Alzheimers Dement. 2019;15(12):1576-1587.

35. Mitchell AJ, Shiri-Feshki M. Rate of progression of mild cognitive impairment to dementia: meta-analysis of 41 robust inception cohort studies. Acta Psychiatrica Scand 2009;119(4):252-265.

36. Richard E, Reitz C, Honig LH, et al. Late-life depression, mild cognitive impairment, and dementia. JAMA Neurol. 2013;70(3):374-382.

37. Dean K, Oulhaj A, Zamboni G, Dejager CA, Wilcock GK. Role of depression in predicting time to conversion to mild cognitive impairment. Am J Geriatr Psychiatry. 2014;22(7):727-734.

38. Bäckman L, Hill RD, Forsell Y. The influence of depressive symptomatology on episodic memory functioning among clinically nondepressed older adults. J Abnorm Psychol. 1996;105(1):97-105.

39. Kumar R, Jorm AF, Parslow RA, Sachdev PS. Depression in mild cognitive impairment in a community sample of individuals 60-64 years old. Int Psychogeriatr. 2006; 18(3):471-480.

40. Geerlings MI, Schmand B, Braam AW, Jonker C, Bouter LM, Van Tilburg W. Depressive symptoms and risk of Alzheimer's disease in more highly educated older people. J Am Geriatr Soc. 2000;48(9):1092-1097.

41. Geerlings MI, Brickman AM, Schupf N, et al. Depressive symptoms, antidepressant use, and brain volumes on MRI in a population-based cohort of old persons without dementia. J Alzheimers Dis. 2012;30(1):75-82.

42. Lin PJ, Daly A, Olchanski N, et al. Dementia diagnosis disparities by race and ethnicity: health services research: cost of care and implications for intervention. Alz heimers Dement. 2020;16:e043183.

43. Gleason CE, Norton D, Zuelsdorff M, et al. Association between enrollment factors and incident cognitive impairment in Blacks and Whites: data from the Alzheimer's Disease Center. Alzheimers Dement. 2019;15(12):1533-1545. 


\section{Neurology}

\section{Predictors of Incident Mild Cognitive Impairment and Its Course in a Diverse Community-Based Population}

Milou J. Angevaare, Jet M.J. Vonk, Laiss Bertola, et al.

Neurology 2022;98;e15-e26 Published Online before print December 1, 2021

DOI 10.1212/WNL.0000000000013017

This information is current as of December 1, 2021

\section{Updated Information \&} Services

References

Citations

Subspecialty Collections

Permissions \& Licensing

Reprints including high resolution figures, can be found at: http://n.neurology.org/content/98/1/e15.full

This article cites 38 articles, 7 of which you can access for free at: http://n.neurology.org/content/98/1/e15.full\#ref-list-1

This article has been cited by 1 HighWire-hosted articles: http://n.neurology.org/content/98/1/e15.full\#\#otherarticles

This article, along with others on similar topics, appears in the following collection(s):

Cohort studies

http://n.neurology.org/cgi/collection/cohort_studies

MCI (mild cognitive impairment)

http://n.neurology.org/cgi/collection/mci_mild_cognitive_impairment Neuropsychological assessment

http://n.neurology.org/cgi/collection/neuropsychological_assessment Risk factors in epidemiology

http://n.neurology.org/cgi/collection/risk_factors_in_epidemiology

Information about reproducing this article in parts (figures,tables) or in its entirety can be found online at:

http://www.neurology.org/about/about_the_journal\#permissions

Information about ordering reprints can be found online:

http://n.neurology.org/subscribers/advertise

Neurology ${ }^{\circledR}$ is the official journal of the American Academy of Neurology. Published continuously since 1951, it is now a weekly with 48 issues per year. Copyright Copyright (C) 2021 The Author(s). Published by Wolters Kluwer Health, Inc. on behalf of the American Academy of Neurology.. All rights reserved. Print ISSN: 0028-3878. Online ISSN: 1526-632X.

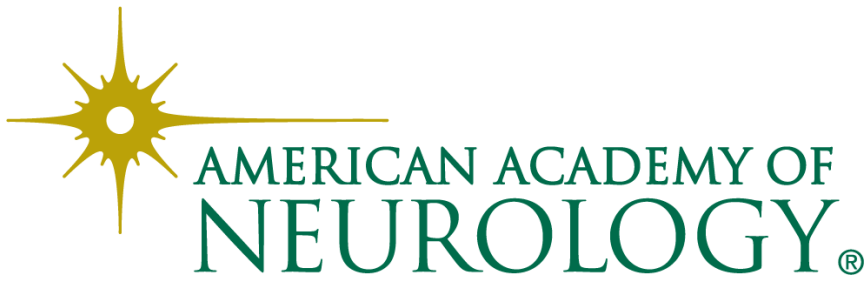

